\title{
A semi-interpenetrating network of polyacrylamide and recombinant basement membrane allows pluripotent cell culture in a soft, ligand-rich microenvironment
}

\author{
Andrew J. Price, Eva Y. Huang, Vittorio Sebastiano, Alexander R. Dunn
}

\begin{abstract}
The physical properties of the extracellular matrix play an essential role in guiding stem cell differentiation and tissue morphogenesis both in vivo and in vitro. Existing work to investigate the role of matrix mechanics in directing stem cell proliferation, self-renewal, and differentiation has been limited by the poor attachment and survival of human pluripotent cells cultured on soft matrices (Young's modulus $\mathrm{E} \lesssim$ $1000 \mathrm{~Pa}$ ). To address this limitation we developed a protocol for generating semi-interpenetrating networks of polyacrylamide and recombinant basement membrane. Using these materials, we found that human embryonic stem cells (hESCs) remained proliferative and pluripotent even when grown in small colonies and on surfaces ranging in stiffness from 150 to $12000 \mathrm{~Pa}$, spanning the range of tissue stiffnesses likely to be encountered in the embryo. Considerable recent attention has focused on the role of the transcriptional coactivator and Hippo effector YAP in regulating differentiation and cell proliferation both in the early embryo and in vitro. We found that while YAP localized to the nucleus on substrates of $E$ $\gtrsim 1000 \mathrm{~Pa}$, its localization was heterogeneous on substrates of moduli $\lesssim 450 \mathrm{~Pa}$, with predominantly nuclear localization at the colony periphery and mixed cytoplasmic and nuclear localization for cells in the colony interior, a pattern reminiscent of YAP subcellular localization in the inner cell mass (ICM) of the early embryo. In addition, hESC colony dynamics were highly responsive to substrate stiffness, with cells assembling into monolayers, multilayer structures, and transient, hollow rosettes in response to decreasing substrate stiffnesses in the range of 12000 to $150 \mathrm{~Pa}$. We suggest that soft, ligand-rich substrates such as are described here provide a promising means of recapitulating aspects of early mammalian development that are otherwise inaccessible, and more broadly may be useful in the derivation of complex tissues from pluripotent cells in an in vitro setting.
\end{abstract}

\section{Introduction}

The ability of pluripotent stem cells to differentiate into any adult cell lineage presents a significant opportunity for scientific study and regenerative medicine. Accordingly, the mechanisms controlling selfrenewal and differentiation of these cells both in vivo and in vitro are of great interest. For differentiated cell types, extracellular substrate stiffness and stress relaxation properties influence cell spreading, proliferation, migration, and matrix invasion [1, 2]. In particular, for mesenchymal stem cells matrix stiffness can influence differentiation, biasing cells on a physiologically soft substrate (E $\lesssim 1000 \mathrm{~Pa})$ toward adipogenesis and those on a physiologically stiff substrate $(E \gtrsim 25000 \mathrm{~Pa}$ ) toward osteogenesis [3]. Substrate stiffness is also found to play a role in enhancing the proliferation and self-renewal of muscle satellite cells [4, 5] and in neural stem cell differentiation [6]. More broadly, additional substrate properties, including topography and extracellular matrix protein spacing, have also been found to influence cellular morphology, organization, and fate specification in a variety of self-renewing populations including mesenchymal stem cells [7, 8], pluripotent cells [9, 10], and epidermal tissues [11].

Current evidence likewise suggests that matrix material properties can strongly influence pluripotent stem cell proliferation, self-renewal, and organoid formation. Substrate stiffness has been shown to influence the morphology and dynamics of colonies of pluripotent cells [12, 13], and the differentiation of pluripotent cells into mesodermal lineages [14]. Recent work demonstrates that colonies of mouse and human pluripotent cells encapsulated in Matrigel, a soft protein hydrogel consisting mainly of basement membrane components, spontaneously form hollow spheres reminiscent of the proamniotic cavity [1518]. However, the underlying mechanisms by which matrix material properties influence pluripotent stem cell differentiation and tissue morphogenesis, either in vitro or in vivo, remain poorly understood.

The Hippo pathway and its downstream transcriptional effector YAP (Yes-associated protein 1) has emerged as an important mechanically responsive signaling pathway involved in tissue development and 
homeostasis [19]. Mechanistically, a variety of studies support an emerging view of YAP as a mechanical signal integrator that regulates cell proliferation in response to cell-cell contact, substrate rigidity, and cell geometry [20]. YAP is likewise thought to play a key role in early embryogenesis. In particular, previous work indicates that YAP plays an essential role in trophectoderm specification [21]. Additionally, stiffnessdependent changes in YAP activity have been associated with differentiation of human embryonic stem cells (hESCs) into neuronal lineages in vitro [22].

The above considerations motivated us to generate simple, robust, and tunable materials that would replicate the physical and biochemical environment present in the early embryo. In contrast to the stiff environment provided by typical in vitro culture conditions, pluripotent cells in the early embryo experience soft, ligand-rich environments. Literature precedent suggests that conventional soft biomaterials, for example common preparations of ECM-functionalized polyacrylamide (PA) hydrogels, are poorly suited for hESC culture. In particular, initial studies found that long term human pluripotent cell attachment and survival on soft 2D microenvironments was poor [23]. Related studies overcame attachment limitations by seeding pre-formed embryoid bodies onto hydrogels early in the process of differentiation [22]. More recent work indicated that the limited attachment of pluripotent stem cells to soft (Young's modulus $\mathrm{E}=400 \mathrm{~Pa}$ ) hydrogels could be improved with the incorporation of multifunctional methacrylates, likely due to an increase in the extracellular matrix (ECM) ligand density on the gel surface $[13,14]$.

To address the limited attachment of pluripotent stem cells to soft materials, we sought to increase the concentration of extracellular matrix protein presented at the substrate surface. Because covalent conjugation of protein to a PA hydrogel is limited by the density of available crosslinking sites at the gel surface, we considered whether an alternative method for protein incorporation might yield soft, $\mathrm{E} \lesssim 1000$ $\mathrm{Pa}$ hydrogels with sufficiently high ligand densities for robust pluripotent cell attachment. Interpenetrating networks (IPNs) present a promising alternative strategy in that they allow separate control of biochemical and physical substrate properties, and have been used successfully with both collagen and Matrigel in alginate for 3D cell culture [24-26]. Interpenetrating materials are generally formed by the sequential or simultaneous polymerization of intermixed precursor solutions, resulting in a polymer networks that are physically interwoven upon polymerization. We therefore hypothesized that an IPN of Matrigel and PA could provide the surface protein densities required for pluripotent cell attachment while allowing independent control of PA hydrogel stiffness.

In this study we describe a semi-interpenetrating matrix of Matrigel and PA and demonstrate its utility for robust culture of hESCs across a wide range in substrate stiffnesses. Consistent with earlier reports, hESCs maintain pluripotency factor expression on semi-IPNs with Young's moduli $\gtrsim 450 \mathrm{~Pa}$ [14]. Building on prior work, we observe that hESC pluripotency, survival, and proliferation are maintained on extremely soft, E $\sim 150 \mathrm{~Pa}$ substrates, even in small colonies of 6-8 cells. Substrate stiffness had a pronounced influence on hESC colony morphology. Clusters of hESCs cultured on E $\sim 12000 \mathrm{~Pa}$ and $450 \mathrm{~Pa}$ surfaces form monolayer and multilayer colonies, respectively. Additionally, on composite substrates with Young's moduli $\lesssim 450 \mathrm{~Pa}$, cells exhibited heterogeneous YAP subcellular localization and a decreased cell proliferation rate in colony interiors, indicative of a role for substrate stiffness and colony geometry on regulating proliferation. Remarkably, colonies cultured on E $150 \mathrm{~Pa}$ substrates exhibited complex morphological rearrangements that included the formation of hollow, polarized rosettes. This process of polarization in pluripotent cells is reminiscent of the formation of the proamniotic cavity, a behavior that has been previously reported for hESCs suspended in 3D Matrigel $[16,17]$. Our findings thus demonstrate that hESCs can replicate the range of structures formed by pluripotent cells in the epiblast (flat sheet), inner cell mass (multicellular mass), and proamnion (hollow sphere) in response to changes in substrate rigidity. We suggest that the ease of use, preparation from commonly available reagents, and tunability make the Matrigel/PA composites described here a potentially useful class of materials for a wide variety of cell biological and tissue engineering applications.

\section{Materials and Methods}


2.1 Preparation of composite substrate. Acrylamide (40\% w/v, Fisher BioReagents), bis-acrylamide ( $2 \% \mathrm{w} / \mathrm{v}$, Fisher BioReagents), and 10X phosphate buffered saline (PBS, pH 7.4, no Mg/Ca, Quality Biological \#119-069-491) stock solutions were diluted in deionized MilliQ-filtered water to yield a polyacrylamide (PA) precursor $2 x$ stock solution of acrylamide and bis-acrylamide in $1 X$ PBS. For this study the 2X PA stock solutions were prepared as follows: (1) E 150 Pa gel: $6 \%$ acrylamide, $0.08 \%$ bisacrylamide, (2) E 450 Pa gel: 6\% acrylamide, 0.12\% bis-acrylamide, and (3) E 12000 Pa gel: 15\% acrylamide, $0.2 \%$ bis-acrylamide. The PA precursor stock was then passed through a $0.2 \mu \mathrm{m}$ syringe filter. If protected from light and stored at $4{ }^{\circ} \mathrm{C}$ the PA precursor solution is stable for several months. Growth Factor Reduced Matrigel (BD \#354230) was provided by the manufacturer at approximately 10 $\mathrm{mg} / \mathrm{mL}$ and was stored in flash-frozen aliquots at $-80^{\circ} \mathrm{C}$. Prior to composite gel preparation, aldehydefunctionalized glass of desired substrate geometry was prepared as previously published [27] with slight modification. In particular, to facilitate the activation of small coverslips, four changes were introduced: (1) fresh coverslips were laid in a single layer on a Petri dish and cleaned and dried by brief exposure to open flame rather than the soap and ethanol washes, (2) solutions were mixed briefly by gently swirling of the Petri dish without continuous stirring, (3) the concentration of 3-aminopropyltrimethoxysilane (APTES) was increased from $2 \%$ to $4 \%(\mathrm{v} / \mathrm{v})$ in $5 \mathrm{~mL}$ of isopropanol, and (4) the concentration of glutaraldehyde was increased from $1 \%$ to $2.5 \%(\mathrm{v} / \mathrm{v})$ in $10 \mathrm{~mL}$ of water. After activation, coverslips can be stored at room temperature, covered and protected from light, for up to two months.

If necessary, Matrigel was diluted in cold Dulbecco's Modified Eagle medium (DMEM; Gibco \#11885-084) to $2 x$ the final desired concentration before the preparation of composite solutions. For composites used in cell culture, which contained a final Matrigel concentration of $5 \mathrm{mg} / \mathrm{mL}$, dilution was not necessary as stocks were provided at $\sim 10 \mathrm{mg} / \mathrm{mL}$. Matrigel-containing solutions must be prepared, thawed, and kept on ice at all times. Matrigel stock and acrylamide/bis-acrylamide solutions were mixed on ice 1:1 to form the composite solution and then placed under vacuum for 20-30 minutes (central building vacuum, $\sim 0.1 \mathrm{~atm}$ ).

Separately, a passivated and hydrophobic surface was prepared by spraying Rain-X onto a clean glass slide. After the excess Rain- $X$ was wiped off with a lint-free tissue and the slide fully dried, the slide was rinsed extensively with deionized MilliQ-filtered water and sonicated for 5-10 minutes (Kendal HB-23MHT sonicator) in a deionized MilliQ-filitered water bath. Rain-X passivated slides were prepared fresh for each gel preparation experiment. Immediately prior to initiation, passivated slides were placed on a metal block on ice in an opened vacuum chamber.

After degassing was complete, freshly prepared ammonium persulfate solution (APS, Acros organics, prepared $30 \% \mathrm{w} / \mathrm{v}$ in water) and tetramethylethylenediamine (TEMED, Fisher BioReagents) were diluted 3:500 and 1:500 by volume, respectively, into the composite solution to initiate polymerization of the PA components. The concentration of APS was increased relative to literature precedent to offset the decreased polymerization rate due to the lowered polymerization temperature [28]. Freshly prepared APS and degassing of all composite solutions were essential to ensure efficient polymerization, as the rate of polymerization is sensitive to temperature and the presence of quenchers that inhibit free-radical catalyzed processes (e.g. oxygen) [28, 29].

Immediately after initiation, the appropriate volume of the composite acrylamide/Matrigel solution required to achieve an average gel thickness of $200 \mu \mathrm{m}$ for the given coverslip geometry (i.e. $100 \mu \mathrm{L}$ for a $22 \mathrm{~mm}$ square coverslip) was pipetted onto the cold Rain-X passivated slide (resting on the metal spacer over ice), and an aldehyde-functionalized coverglass was carefully inverted onto the droplet. The polymerization reaction was placed under vacuum to prevent condensation of water on or near the polymerizing composite solutions. An optional additional step of backfilling the vacuum chamber with a sub-atmospheric pressure of dry nitrogen ( $\sim 0.9 \mathrm{~atm})$ during polymerization, while not employed in the materials generated for this study, was subsequently found to reduce the appearance of bubble defects without inhibiting polymerization. The polymerization reaction was incubated for exactly 30 minutes after the establishment of vacuum. After polymerization of the polyacrylamide, the solution was placed in a humidified $37^{\circ} \mathrm{C}$ incubator for 30 minutes to induce gelation of the Matrigel. The coverslip with the 
polymerized composite was then carefully removed from the glass slide, inverted into a cell culture vessel, and equilibrated in DMEM supplemented with Pen/Strep (Gibco 15140-122) overnight before cell seeding, rheometry, or staining experiments.

2.2 Cell culture. Human Embryonic Stem cells (WA09) were maintained in a feeder-free cell culture system (Essential 8 medium, ThermoFisher A1517001) supplemented with penicillin-streptomycin (ThermoFisher 15140122) and cultured in a humidified incubator with $5 \% \mathrm{CO}_{2}$ at $37^{\circ} \mathrm{C}$. Cells were regularly maintained on Matrigel-coated tissue culture plastic vessels and subcultured at a 1:8 splitting ratio as small clumps by treatment with $0.5 \mathrm{mM}$ ethylenediaminetetraacetic acid (EDTA, ThermoFisher AM9261) in Dulbecco's phosphate-buffered saline (DPBS, Corning 21031CV). For experiments requiring cell recovery from composite gels, cells were seeded onto and sub-cultured from the composite gel with Accutase (Innovative Cell Technologies). During the first 24 hours after splitting with Accutase, or if vigorous pipetting of EDTA-treated cells resulted in excessive dissociation, $10 \mu \mathrm{M}$ Y-27632 (Santa Cruz Biotechnology, sc-216067) was added to the cell culture medium to enhance the survival of single cells [30]. All staining and timelapse experiments were performed at least 24 hours after the withdrawal of $Y$ 27632 from cell culture medium, a sufficient amount of time for colony morphology to re-equilibrate (Figure S1).

2.3 Rheometry. Due to the relatively high reagent cost and the number of replicates required to ensure a well-characterized stiffness measurement, a small, $3 \mathrm{~mm}$ probe was used for the rheological characterization of matrix stiffness. An AR-G2 rheometer was fitted with a $3 \mathrm{~mm}$ steel parallel plate probe for all rheology measurements. Composite gels were prepared as described; after overnight equilibration in DMEM, gels were washed $1 \mathrm{x}$ with PBS and placed on the rheometer platform. The rheometer platform temperature was fixed at $37^{\circ} \mathrm{C}$ after placement of the probe over the center of the $\sim 200 \mu \mathrm{m}$ thick gel (prepared on a $12 \mathrm{~mm}$ diameter, \#2 thickness coverslip). A $100 \mu \mathrm{L}$ droplet of PBS was added to the gel surface to ensure constant hydration during the measurement. The mechanical properties of each substrate (G' and G") were measured with a strain sweep from $0.1 \%$ to $100 \%$ oscillatory shear strain at $0.25 \mathrm{~Hz}$.

2.4 Immunostaining and Microscopy. For immunostaining experiments, cells and surfaces were fixed with freshly prepared 4\% paraformaldehyde diluted in PBS (pH 7.4). Fixation was performed for 15 minutes at room temperature, and samples were washed with PBS and stored at $4{ }^{\circ} \mathrm{C}$ until staining. For immunostaining, samples were blocked and permeabilized with $1 \%$ bovine serum albumin in PBS $+0.1 \%$ (v/v) Triton-X100 for 1-2 hours at room temperature. Primary antibodies were diluted into blocking buffer and incubated with samples overnight at $4{ }^{\circ} \mathrm{C}$ with gentle rocking. Primary antibodies were used at the following dilutions: rabbit anti-Collagen-IV (Abcam ab6586) 1:500, mouse anti-E-Cadherin (Abcam ab1416) 1:100, rabbit anti-Laminin (Sigma PA1-16730) 1:500, rabbit anti-Nanog (Abcam ab109250) 1:500, mouse anti-Oct4 (Santa Cruz sc-5279) 1:200, rabbit anti-Oct4 (Cell Signaling 2750S) 1:400, mouse anti-Sox2 (R\&D Systems MAB2018) 1:500, mouse anti-YAP (Novus Biologics 2F12) 1:200, and rabbit anti-YAP (Cell Signaling 4912S) 1:200. After overnight primary staining, samples were washed three times with PBS and stained for 1.5-2 hours at room temperature with a 1:1000 dilution of fluorescently labelled anti-Mouse FAB2 (Cell Signaling Alexa488 - 4408S, Alexa555 - 4409S, or Alexa647 - 4410S), a 1:1000 dilution of fluorescently labelled anti-Rabbit FAB2 (Cell Signaling Alexa488 - 4412S, Alexa555 - 4413S, or Alexa647 - 4414S), a 1:100 dilution of Alexa-647 phalloidin (Cell Signaling 8940S), and/or 0.5-1 $\mu \mathrm{g} / \mathrm{mL}$ Hoechst 34580 (ThermoFisher) diluted in blocking buffer. For protein quantification within composite gels, gels were fixed with $4 \%$ paraformaldehyde, washed twice in PBS $+0.1 \% \mathrm{v} / \mathrm{v}$ TritonX-100, and then stained with the Krypton Orange fluorescent protein stain (ThermoFisher \#46628) according to the manufacturer instructions. Epifluorescence microscopy for immunostaining quantification and time-lapse videos were performed on a Nikon Ti-E microscope controlled with Micromanager 1.4.20. Confocal and AiryScan [31] acquisitions were performed with a Zeiss LSM 780 scanning confocal microscope. 
2.5 Sequential substrate passaging. To ensure a large enough cell sample size for mRNA and immunofluorescence quantification we used $22 \mathrm{~mm}$ square coverslips placed in a 6-well tissue culture vessel for sequential passage experiments. Each hydrogel was cultured in duplicate. To prevent cell attachment to the tissue culture plastic, hydrogel-covered coverslips were placed into a clean, uncoated six well dish and cells were seeded directly onto a DMEM-equilibrated hydrogel in a 900-1000 $\mu \mathrm{L}$ droplet. Careful handling ensured the cell/medium droplet remained on the hydrogel and did not spread onto the surrounding tissue culture plastic. After attachment overnight, medium was changed daily with $2 \mathrm{~mL}$ of Essential 8 medium covering each well. As an additional precaution, coverslips were removed to a second clean well before Accutase treatment to ensure that all recovered cells came from the hydrogel surface. For passaging, we found that EDTA alone was not sufficient to efficiently detach pluripotent cells that had been cultured on composite hydrogels. Instead, we found that treatment with Accutase for 10 minutes, careful cell detachment with a cell scraper, and mixing by pipette could effectively remove cells from the substrate.

During passaging, $35 \%$ to $50 \%$ of the cells recovered after four days of culture were seeded onto new hydrogels to achieve approximately a 1:6-1:8 dilution of cells. Additional cells were seeded into Matrigelcoated, eight-well coverslip chambers (ThermoFisher \#155409) for immunostaining of pluripotency markers. The remaining cells were used for mRNA extraction for GPCR evaluation. At the conclusion of the experiment, all samples were immunostained in parallel and imaged on the same day with identical imaging parameters, and each mRNA sample was reverse transcribed, mixed, and quantified within the same 2 day period at the conclusion of the experiment.

2.6 Quantitative Immunofluorescence. To allow for automated nuclear segmentation, cells from each condition and time were sub-cultured onto a Matrigel-coated eight well coverslip chamber for two days prior to fixation (schematic in Figure S5). Fixed samples were stored at $4{ }^{\circ} \mathrm{C}$ until the end of the experiment, when all samples were immunostained in parallel for the pluripotency factors Oct4, Sox2, and Nanog. Images for each condition were taken with a 20x Plan Apo objective (NA 0.75) by an automated multi-channel acquisition function in Micromanager 1.4.20, and the in-focus frame was chosen by the built-in software autofocus on the nucleus (Hoechst 34580) channel. After image acquisition, nuclei in the Hoechst (DNA) channel were detected by CellProfiler software [32] and segmented using the automatic threshold strategy. The nuclei mask was then applied to other channels to obtain the mean fluorescence intensity for each nuclear region. A Matlab script was used to normalize the mean fluorescence intensity by the mean value of the segmentation channel (Hoechst) to correct for illumination variation. The corrected intensities were further normalized against the mean value for the Day 4 glass control to determine the relative changes in nuclear protein stain between the different conditions.

2.7 Quantitative PCR. Cellular mRNA samples were extracted with the GeneJet RNA Purification Kit (ThermoFisher $\mathrm{K} 0731$ ) and stored at $-20^{\circ} \mathrm{C}$ until the conclusion of the experiment. Reverse transcription to cDNA was performed with the Applied Biosystems High Capacity cDNA Reverse Transcription Kit with RNAse Inhibitor (ThermoFisher \#4374966) and evaluated on a StepOnePlus with either the Power SYBR Green PCR Master Mix (ThermoFisher \#4367659) or the PowerUP SYBR Green PCR Master Mix (ThermoFisher \#A25742) - the change in sensitivity observed between these kits did not significantly impact the results when normalized against the housekeeping gene GAPDH. All kits were used according to the manufacturer instructions.

2.8 EdU staining and analysis. EdU labeling was performed with a ClickiT PLUS Alexa-647 Imaging Kit (ThermoFischer \#C10640) according to the manufacturer instructions. Sample blocking and immunostaining was performed immediately after the Click conjugation of Alexa-647. Immunolabeling incubations were all protected from light to prevent photobleaching of the Alexa-647, but otherwise the staining protocol was unchanged.

Automated nuclear segmentation with CellProfiler was unable to accurately segment individual nuclei due to the compactness of pluripotent stem cells on soft substrates, with densely packed nuclei in three dimensions. To quantify the EdU incorporation within individual nuclei, the center of each nucleus was 
manually identified in the plane of maximal area for that nucleus. From these centroids a custom MATLAB code was used to segment a $6 \mu \mathrm{m}$ diameter circle around the point of interest including the two adjacent confocal slices ( $2 \mu \mathrm{m}$ above and below) and evaluate an average intensity in the YAP, Hoechst (DNA), and EdU channels. For the EdU channel, a local background subtraction (60 $\mu \mathrm{m}$ in size) and absolute contrast adjustment (saturating $0.1 \%$ of pixel values) were required, and EdU positive nuclei were identified as those at least three times above the background intensity of EdU-negative nuclei. The automated binary selection agreed well with visual binary classification in all conditions. EdU staining samples were prepared in biological triplicate, and three positions on each gel were imaged with confocal microscopy.

Due to variation in the absolute YAP immunolabelling intensity for cells on composite substrates (Figure 7A) the average cytoplasmic intensity for each condition was determined by sampling a cytoplasmic area for each of the nine positions using Fiji/lmageJ. From this average cytoplasmic intensity, nuclei were classified as "nuclear YAP" if they were at least 1.1 times the average cytoplasmic intensity, and "cytoplasmic YAP" if they were less than 0.9 times the cytoplasmic value. The remaining nuclei were classified as "equivalent."

2.9 Statistical analysis and bootstrap model. Tests for significant changes in mean stiffness, mean intensity, or mean Pearson's correlation coefficient were performed with a two-sample, two-tailed unequal variance t-test. For EdU staining analysis, the statistical uncertainty of the measured EdU incorporation rate was evaluated using the Bootstrap algorithm [33], which we implemented using a custom Matlab code. The error on the mean proliferation rate was estimated from $10^{6}$ simulated measurements. The probability that two conditions had an equivalent mean proliferation rate was estimated from the overlap of their simulated parameter distributions.

\section{Results}

\subsection{Sequential polymerization and gelation of polyacrylamide and Matrigel results in a composite substrate of tunable stiffness.}

In this study we sought to address the poor attachment of pluripotent stem cells to soft materials by increasing the surface density of the extracellular matrix ligand, which we reasoned might promote cell attachment and survival. Polyacrylamide (PA) has been widely used in biomechanical studies due to its highly tunable mechanical properties. However, surface functionalization with cell-binding ligands is required for cell attachment and culture on PA hydrogels. A variety of methods have been employed to conjugate functional peptides onto PA hydrogels [12, 27, 34-36]. However, the maximum concentration of peptides that can be covalently attached to the gel surface depends on the number of available network attachment sites on the surface of a PA hydrogel, which in turn depends on the acrylamide concentration. Thus, current strategies for functionalizing PA matrices are sensitive to the decreases in acrylamide concentration needed to generate soft matrices.

To potentially circumvent this difficulty, we developed a method to incorporate an interpenetrating matrix of recombinant basement membrane (in this study, Matrigel) into a PA hydrogel by sequential polymerization, first of the polyacrylamide, and second of the Matrgiel by gelation via thermoswitching from $0{ }^{\circ} \mathrm{C}$ (wet ice) to $37^{\circ} \mathrm{C}$ (Figure 1). We investigated three formulations of acrylamide and bisacrylamide with four concentrations of Matrigel ranging from $0 \mathrm{mg} / \mathrm{mL}$ to $5 \mathrm{mg} / \mathrm{mL}$. The resulting composite materials were characterized by bulk rheology to determine composite matrix shear stiffness and the effect of Matrigel, if any, on composite stiffness. In the absence of Matrigel, using a DMEM control, the measured shear modulus of the three PA formulations were $\mathrm{G}=71 \pm 9 \mathrm{~Pa}$ (standard error on the mean, $\mathrm{N}=15), 151 \pm 6 \mathrm{~Pa}(\mathrm{~N}=8)$, and $4250 \pm 250 \mathrm{~Pa}(\mathrm{~N}=8)$.

Due to the fragility of Matrigel alone, the stiffness of the Matrigel without any PA components was measured with fewer replicates and with gelation of the Matrigel precursor directly under an 8-mm parallel plate geometry. The shear modulus of Matrigel was found to be $\mathrm{G}=23 \pm 3 \mathrm{~Pa}(\mathrm{~N}=3)$ at $5 \mathrm{mg} / \mathrm{mL}$, the 
concentration used in cell culture experiments (Figure S9). Thus, the relative stiffness of the underlying Matrigel network was expected to be significantly softer than the stiff PA hydrogel, more than six times softer than the intermediate PA hydrogel, and approximately three times softer than the softest PA hydrogel.

Consistent with this reasoning, shear rheometry measurements showed that the mechanical properties of the composite materials appeared to be dominated by the parent polyacrylamide network (Figure 2). The full strain-elasticity relationships - plotted in log space - showed almost complete overlap between all measured Matrigel concentrations for each PA formulation. In the softer materials, strains below 5\% induced torques that were too low to reliably measure. For this reason, the storage modulus at $10 \%$ strain was chosen to estimate the material stiffness in the linear elastic regime. A frequency sweep from $0.1 \mathrm{~Hz}-$ $10 \mathrm{~Hz}$ determined that the measured elastic response was most consistent for measurements below $\sim 1$ $\mathrm{Hz}$; accordingly, all elasticity measurements were performed at $0.25 \mathrm{~Hz}$ to determine the approximate stiffness in the low-frequency limit.

In this regime we found that the shear storage modulus ( $\left.G^{\prime}\right)$ of all measured composite hydrogels exceeded the measured shear loss modulus (G"), by approximately two orders of magnitude, indicating an elastic strain response with minimal viscous dissipation. Instrument noise in the low-strain regime made quantitative inferences of viscoelastic trends difficult. In the regime where measurements were not limited by instrument noise, we found that the storage modulus of the softest composite matrix formulation decreased from $\mathrm{G}=71 \pm 9 \mathrm{~Pa}(\mathrm{~N}=15)$ to $\mathrm{G}=54 \pm 7 \mathrm{~Pa}(\mathrm{~N}=15)$ in the presence of $5 \mathrm{mg} / \mathrm{mL}$ of Matrigel (Figure 2B). The storage moduli of the intermediate and stiff materials, in contrast, did not change significantly in the presence of $5 \mathrm{mg} / \mathrm{mL}$ Matrigel, with measured shear moduli $\mathrm{G}=149 \pm 32 \mathrm{~Pa}$ $(\mathrm{N}=8)$ and $4250 \pm 250 \mathrm{~Pa}(\mathrm{~N}=8)$ respectively. In all of the following cell culture and staining experiments the concentration of Matrigel incorporated into the composite matrix was fixed at $5 \mathrm{mg} / \mathrm{mL}$.

For clarity with respect to literature precedent, subsequent sections refer to the composite gel by their approximate Young's modulus (E). The Young's modulus $(E)$ is related to the shear modulus $(G)$ by a factor that depends on the Poisson ratio $(v)$ of the material: $E=2^{*} G(1+v)$ [37]. Here we assumed the bulk mechanical properties of the composite matrix to be largely dominated by the PA network, implying a Poisson ratio in the range of $v \sim 0.45-0.5$ [38]. Thus the three formulations prepared with $5 \mathrm{mg} / \mathrm{mL}$ of Matrigel - with shear moduli $\mathrm{G}=54,149$, and $4150 \mathrm{~Pa}$ - can be referred to by their approximate Young's moduli of $\mathrm{E} \sim 150,450$, and $12000 \mathrm{~Pa}$.

\subsection{Soft composite hydrogels present sufficient surface ECM ligands for robust pluripotent cell attachment.}

We employed several strategies to visualize the distribution of protein within the composite materials, $i$ ) immunostaining for collagen IV and laminin, ii) imaging tetramethylrhodamine (TMR)-functionalized Matrigel incorporated into the PA/Matrigel composite matrix, or iii) staining for total protein with the nonspecific protein stain Krypton Orange (Figure 3, Figure S2). Of these techniques, we found Krypton Orange to be useful due to its large and linear dynamic range and its ability to effectively label the interior of the hydrogel.

The surfaces of composite PA/Matrigel matrices showed both an approximately uniform background level of protein staining along with apparent networks of protein fibers that were more widely spaced for substrates with lower elastic moduli (Figure 3A). For reference, the glass control surface showed punctate clusters of protein distributed randomly over the surface, indicative of the heterogeneous adsorption of diluted Matrigel from solution at concentrations sufficient for feeder-free pluripotent cell culture [39]. The relative amount of protein at the surface of the $5 \mathrm{mg} / \mathrm{mL}$ composite substrates determined by laminin or Krypton Orange stain was approximately the same as or two-fold less than the amount adsorbed onto the surface of our glass control preparation regularly used for cell culture. (Figure 3B). 
To better understand the material properties of the composite, we employed a super-resolution imaging technique, AiryScan imaging, to visualize the sub-micron organization of the protein component within the gel. In the interior of the composite matrixes, the Matrigel protein component was partially localized to approximately circular inclusions that increased in size from less than $0.5 \mu \mathrm{m}$ to more than $1 \mu \mathrm{m}$ in diameter with decreasing hydrogel stiffness (Figure 3C). However, appreciable fluorescence signal in the regions between these protein-dense regions indicated that Matrigel likely extended throughout the gel. Direct visualization of the polyacrylamide network within the composite PA/Matrigel hydrogel via electron microscopy was not possible due to the sensitivity of soft hydrogels to the sample drying necessary for scanning electron microscopy. However, the lack of a significant change to the bulk stiffness of the PA network upon the addition of Matrigel suggests that its material properties were minimally altered, implying that the PA network was also continuous. Given these results, we describe the material as a semi-IPN rather than a complete IPN.

An important requirement for our PA/Matrigel semi-IPN was that the protein network remained stable for multiple days under cell culture conditions. Accordingly, we characterized the stability of Matrigel incorporated into the hydrogel in cell culture conditions via two complementary methods. Quantification of surface protein with Krypton Orange was performed for gels incubated for one, three and five days in cell culture medium in a humidified, $37^{\circ} \mathrm{C}$ cell culture incubator supplemented with $5 \% \mathrm{CO}_{2}$. We found that the protein structure on the composite gel surface remained largely stable (Figure S8A). A modest decrease in surface protein, as quantified using Krypton Orange staining, was observed in the glass control, intermediate PA, and soft PA conditions with five-day incubation, suggesting that a subset of proteins at the surface were mobile (Figure S8B). In all cases, at least $60 \%$ of the original staining intensity was retained after five days. As a complementary method to measure total protein loss from the hydrogel, we quantified the protein lost to solution in each day via Bradford Assay. After an initial loss of protein during the first overnight incubation, little additional protein loss was observed, again suggesting that the gel composition remained stable over the course of our experiments. (Figure S8C).

\subsection{Substrate properties instruct colony morphology and alter YAP localization.}

To evaluate the response of human embryonic stem cells to these soft substrates, we passaged cell clusters from tissue culture plastic directly onto DMEM-equilibrated composite hydrogels (see Methods 2.2, Figure 4). Consistent with published work, we found that colonies adopted an altered morphology [12] and exhibited decreased nuclear localization of the transcriptional co-activator YAP [23] on soft composite substrates (Figure 5). In addition, we found that the localization of YAP varied significantly within a single colony on substrates with elastic moduli $\mathrm{E} \sim 150$ and $450 \mathrm{~Pa}$, with heterogeneous localization of YAP in the colony interior and increased nuclear localization on the colony exterior (Figure 5A). The possible role of YAP as a local cue in hESC colonies is intriguing due to the developmental role of YAP as a positional cue in mouse development to distinguish cells on the exterior of the embryo, which form the trophectoderm, from those in the interior, which form the inner cell mass [21, 40, 41]. Nuclear YAP also acts as an enhancer of the naïve state in human pluripotent cells, and its role as a positional cue in the human blastocyst may differ appreciably from mouse [42].

Reducing matrix stiffness from $\mathrm{E} \sim 450 \mathrm{~Pa}$ to an extremely soft $\mathrm{E} \sim 150 \mathrm{~Pa}$ changed the overall structure of colonies to a more spherical shape (Figure 4A), suggesting that the cell colonies were responsive to this change in mechanics. However, the heterogeneous localization of YAP did not change significantly from the $450 \mathrm{~Pa}$ surface to the $150 \mathrm{~Pa}$ surface, suggesting that in the extremely soft limit, the differential localization of YAP to the nucleus in a subset of cells may be due to some factor other than bulk matrix stiffness. Consistent with this hypothesis, imaging of embryoid bodies - clumps of pluripotent cells cultured in solution - demonstrated a similar variation in YAP nuclear localization after four days of culture in the absence of any matrix (Figure S3).

The altered YAP localization induced in pluripotent stem cells on soft materials was, importantly, distinct from its regulation at the transcriptional level: pluripotent cells cultured on soft substrates did not show a significant loss of YAP mRNA, and nuclear YAP localization was recovered within two days of attachment 
to a glass coverslip (Figure S6). In contrast, treatment of human embryonic stem cells with $20 \mu \mathrm{M}$ verteporfin, a small molecule inhibitor of the YAP-TEAD interaction, caused cell death within 24 hours (Figure S7). Together these data suggest that a basal level of YAP/TEAD transcriptional activity, which may be required for cell survival, was present even in cells cultured on soft materials.

As noted, pluripotent cell clusters cultured for five days on the $\mathrm{E} \sim 150 \mathrm{~Pa}$ substrate adopted a curved spheroidal shape, while those on stiffer materials formed monolayers (Figure 4). To better understand how these colony shapes arose we performed a time-lapse microscopy experiment to observe colony shape during cell growth (Video S1). On the softest substrate, E 150 Pa, we observed dynamic variation in colony shape through the course of our experiment, while growth on the stiffer $\mathrm{E} \sim 12000 \mathrm{~Pa}$ substrate was qualitatively similar to the glass control. In particular, when pluripotent cell clusters on the $E$ $\sim 150 \mathrm{~Pa}$ substrate approached $\sim 50 \mu \mathrm{m}$ in diameter, a ring of increased cell density formed at the outside edge of the colony, surrounding a central disk of cells (Figure 4C, Video S1A). This transient phenotype did not persist as cell clusters grew beyond $\sim 100 \mu \mathrm{m}$ in diameter, indicating an interplay between matrix properties, colony size, and colony shape.

Although seeding clump size was difficult to control precisely, seeding small ( 4-8 cell) EDTA-dissociated clumps uncovered additional phenomena, including rosette formation and hollowing structures that were reminiscent of those found in isolated epiblast cells and pluripotent cells grown in Matrigel (Figure S4) $[15,17,18]$. Possibly due to the difficulty of obtaining a cell clump that was small enough to polarize efficiently but large enough to allow survival without the contractility inhibitor $Y-27632$, such structures were found only in a subset of colonies on the softest $\mathrm{E} \sim 150 \mathrm{~Pa}$ composite material, specifically colonies with fewer than $\sim 32$ cells after two days of culture. Further, only half (four of eight) of colonies with less than 32 cells in this condition exhibited hollow lumens when fixed at 48 hours post seeding. Hollow structures were not found in a parallel substrate cultured for two additional days (four days total), suggesting that they were likely transient.

\subsection{Composite matrixes of PA and Matrigel support pluripotent stem cell self-renewal.}

Pluripotency maintenance in embryonic stem cells is most strongly associated with the expression of a network of three transcription factors, Oct4, Sox2, and Nanog [43]. It is not established whether soft substrates can induce differentiation of human embryonic stem cells in the absence of other cues. To address this question we sought to characterize the maintenance of these factors at the transcriptional level using quantitative PCR (qPCR), while in parallel monitoring their nuclear localization with immunofluorescence. After approximately 4-5 days of culture on all substrates, cell colonies became large and approached confluency (Figure S1). For this reason, the longest timepoint evaluated without sub-culturing was five days (Figure $\mathbf{6 A}$ ); to address this limitation we developed a protocol to extract and subculture cells from the composite hydrogel (see Methods 2.5).

To evaluate the possibility of a gradual and irreversible stiffness-induced differentiation, cells on composite hydrogels for the indicated interval (4 or 8 days) were harvested, seeded onto Matrigel-coated coverslips, and maintained under regular cell culture conditions for two days prior to fixation. Individual nuclei were segmented and quantified for Oct4, Sox2, and Nanog immunofluorescence stains (Figure 6B, D, F). We found little change in the nuclear intensity of these factors after four and eight days across a wide substrate stiffness range. In addition, the $1^{\text {st }}$ percentile (low) whisker remained near or above 0.5 in all cases, indicating that no significant subset of cells showed signs of differentiation. An experiment extending the culture for two additional passages, 16 days on the composite hydrogels, also showed no significant loss of these factors (Figure S5).

In parallel, qPCR experiments were performed on mRNA collected directly from cells detached from the hydrogel, providing an immediate measure of transcriptional changes of POU5F1, SOX2, and NANOG due to stiffness variations. Results from the GPCR performed on hydrogel and control samples showed that the fold-change in mRNA for POU5F1, SOX2, and NANOG remained within a factor of two of the glass control (Figure 6C, E, G). The most variable transcription factor at the mRNA level was NANOG, 
with changes in NANOG mRNA levels apparent in many of the day 4 and day 8 samples. However, similar variation was also observed in the glass control. The overall maintenance of these pluripotency factors indicated that the heterogeneous localization of YAP induced by the soft composite substrates was not sufficient to cause an appreciable loss of these pluripotency markers.

\subsection{Proliferation rate is spatially variable in pluripotent cell colonies cultured on soft materials}

After determining the minimal effect of soft microenviroments on human embryonic stem cell pluripotency maintenance, we sought to precisely quantify the effect of soft substrates on YAP localization and proliferation rate. The thymidine analog 5-Ethynyl-2'-deoxyuridine (EdU) labels cells in the synthesis (S)phase by its incorporation into the DNA during genome replication. In our experiments, the EdU treatment window -30 minutes - was short relative to the cell cycle timescale ( $>10$ hours) and thus acted as a binary label of the subset of cells in S-phase (Figure 7A). The results of the EdU labeling (Figure 7B, C) demonstrated a small but significant decrease in the average frequency of DNA incorporation on soft composite substrates. After four days of growth on composite PA/Matrigel substrates, $42 \%$ of cells incorporated EdU on the softest composite $(\mathrm{E} \sim 150 \mathrm{~Pa})$. This value was high relative to literature values for other cell types, but significantly lower than the $61 \%$ measured for pluripotent cells grown on glass ( $p$ $<0.001$ ). Between day 2 and day 4, the relative error decreased substantially due to the increased number of nuclei from cell growth, but the rate of EdU labeling for the E $\sim 150 \mathrm{~Pa}$ gel, the $\mathrm{E} \sim 450 \mathrm{~Pa}$ gel, and the glass control was not significantly changed between these time points $(p>0.05)$. The stiff $E \sim$ $12000 \mathrm{~Pa}$ gel condition registered a slight increase in average proliferation rate from $53 \%$ at day 2 to $58 \%$ at day $4(p=0.042)$.

The multilayered morphology found at $\mathrm{E} \sim 150 \mathrm{~Pa}$ and $450 \mathrm{~Pa}$ was associated with a decrease in EdU incorporation for cells in the interior relative to the exterior of the colony. For simplicity, the middle slice of each colony was segmented into exterior nuclei (within $10 \mu \mathrm{m}$ of the edge) and interior nuclei inside of this shell. Cells on both the $\mathrm{E} \sim 150 \mathrm{~Pa}$ and $450 \mathrm{~Pa}$ composite substrates showed a significant difference in EdU incorporation $(p<0.01$ ) between the exterior and the interior (Figure 7D). On the $\mathrm{E} \sim 150 \mathrm{~Pa}$ composite substrate the interior nuclei incorporated EdU at a rate of 0.66 relative to the exterior nuclei, and on the $\mathrm{E} \sim 450 \mathrm{~Pa}$ composite the interior nuclei incorporated $\mathrm{EdU}$ at a rate of 0.75 relative to the exterior.

Due to variation in the absolute YAP immunolabeling intensity for cells on composite substrates (Figure 7A), the intensity within each selected nucleus was classified against the average cytoplasmic value for that condition. Nuclei with an average YAP intensity $<0.9$ of the average cytoplasmic value were classified as "cytoplasmic YAP", while those $>1.1$ of the average cytoplasmic value were classified as "nuclear YAP," and the remaining nuclei were classified as "equivalent YAP". From this analysis we found that the fraction of cells with cytoplasmic YAP increased between day 2 and day 4 of culture (Figure 7E, F). A potential confounding factor responsible for the increased cytoplasmic YAP localization after two additional days of culture on the soft $\mathrm{E} \sim 150 \mathrm{~Pa}$ and $450 \mathrm{~Pa}$ substrates was the size of the cell colonies. After two days the average number of cells in each colony was approximately 30 on the $\mathrm{E} \sim 150 \mathrm{~Pa}$ and $\mathrm{E}$ $\sim 450 \mathrm{~Pa}$ substrates. This increased to approximately 200 nuclei per colony in the $\mathrm{E} \sim 150 \mathrm{~Pa}$ and $\mathrm{E}$ $450 \mathrm{~Pa}$ substrates after four days. As the spheroidal colonies on $\mathrm{E} \sim 150 \mathrm{~Pa}$ and $450 \mathrm{~Pa}$ substrates grew, the number of cells in the center of the cell colony increased, which correlated with a decrease in nuclear YAP localization (Figure 7E, F). However, the level of compaction in each individual colony was not correlated with proliferation rate (Figure 7G) and the overall fraction of cells that were positive for EdU incorporation did not change significantly between the day 2 and day 4 timepoints for the $\mathrm{E} \sim 150 \mathrm{~Pa}$ and $450 \mathrm{~Pa}$ substrates. Together these data imply that reduced nuclear localization of YAP on soft materials did not induce cell cycle arrest, though it was indirectly correlated with a decreased proliferation rate.

\section{Discussion}

A semi-interpenetrating composite of Matrigel and PA presents a useful material for investigating cellular behavior in soft microenvironments where cell attachment is limited by surface ligand concentration. These composite matrixes allow for a tunable mechanical stiffness, determined largely by the PA hydrogel, while retaining the dense presentation of cell-binding Matrigel proteins that appears to be 
required for adherent hESC culture. Using this composite substrate we found robust adhesion and growth of hESCs, sufficient for sequential passages, on a wide range of material stiffnesses, including extremely soft E 150 Pa surfaces.

Although the elastic modulus is likely to play a dominant role in influencing the response of hESCs to various Matrigel/PA compositions, an influence of factors that co-vary with stiffness cannot be ruled out. Matrix pore size and concomitant alterations in protein tethering and spacing have been identified as control parameters for cell behavior in mechanobiological studies of differentiation [7]. It is also possible that variations in the concentration of retained Matrigel across gels of different stiffness may influence hESC behavior. However, Matrigel concentrations remained either unchanged or decreased less than 0.5-fold across an 80-fold decrease in stiffness (Figure 3), suggesting that differences in ligand availability are less likely to account for our observations. Finally, pluripotent cell deposition of collagen IV and remodeling of laminin were observed after several days of culture, and could conceivably occur in a stiffness-dependent manner (Figure S2). Thus, although the materials used in this study are referred to by their approximate Young's moduli for simplicity, other properties of the composite PA/Matrigel substrates can in principle influence cellular phenotype.

Consistent with other studies we find that hESCs maintain pluripotency factor expression on substrates with stiffness greater than E 450 [14]. Here we extend these observations by showing that pluripotency, survival, and proliferation can be maintained on an E 150 Pa substrates, even in small colonies of 6-8 cells. In addition, we find that the heterogeneous localization of YAP induced by a soft environment is not sufficient to cause a loss of pluripotency. The contrast between pluripotent stem cell survival on soft materials and cell death under verteporfin treatment (Figure S7) supports a provisional model in which multiple regimes of YAP activity are responsible for survival, proliferation, and differentiation. Under this model, pluripotent stem cells maintain a basal level of YAP activity sufficient for proliferation and survival even in soft microenvironments, yet may also relocalize additional YAP to the nucleus in response to an activating stimulus. In agreement with this model, although YAP-dependent transcriptional regulation is required for trophectoderm specification [21, 40, 41, 44], YAP exclusion from the nuclei of human ICM cells is not complete [42], perhaps because these cells must continue to proliferate for development to proceed. Together these results imply that a precisely tuned level of YAP signaling in response to the cell microenvironment can allow both mechanosensation and cell survival and proliferation.

Recent studies have revealed that colony size, cell position within a colony, and the properties of the extracellular matrix can exert strong influences on pluripotent stem cell differentiation. Spatial confinement of pluripotent cell colonies onto patterned disks is found to induce an organized, circumferential pattern of differentiation into ectodermal, mesodermal, and endodermal lineages upon exposure to BMP4 [10]. Complementary investigations find that patterned, 3-mm sized hESC colonies on soft $E=1000 \mathrm{~Pa}$ substrates exhibit localized cell-ECM traction forces around the edge of the colony [13]. Additionally, changes in $\beta_{1}$ integrin activation have been found to potentiate pluripotent stem cell differentiation into mesenchymal lineages [14]. In our study we find that proliferation rate, in addition to YAP localization, varies with cell position within hESC colonies cultured on soft materials (E $\lesssim 450 \mathrm{~Pa}$ ). Thus, both our and previously published observations suggest that physical inputs such as geometry, substrate stiffness, and ECM composition can exert long-range control over cell differentiation, proliferation, and tissue patterning in pluripotent stem cell models.

We observe a shift in hESC colony morphology from monolayer, to multilayer, and finally to transient rosette formation in response to decreasing substrate stiffness. Consistent with our findings in the extremely soft regime, previous work has found that human pluripotent stem cells encapsulated in a soft Matrigel matrix spontaneously form hollow spheroids, a process reminiscent of the formation of the proamnionic cavity [17]. In the embryo, epiblast cells at the interface of the proamniotic cavity and the primitive endoderm subsequently undergo gastrulation to establish the germ layers [45]. We speculate that the approaches presented here may, with some modification, be useful for constructing models of the 
morphogenetic movements and fate specification events to reveal essential aspects of early mammalian development that are otherwise inaccessible in an in vivo setting.

\section{Acknowledgements}

We thank Emily Hollenbeck and Gerald Fuller for advice, training, and access to the AR-G2 rheometer used for sample rheology. We thank the Stanford Institute for Stem Cells and Regenerative Medicine for extensive help and training. Confocal imaging was performed in the Stanford Cell Sciences Imaging Facility with a Zeiss LSM 780 funded jointly by the Stanford School of Engineering and the Beckman Center (Alex Dunn and Jon Mulholland, PIs). This work was funded by the National Science Foundation Graduate Research Fellowship (A. J. P.), the Stanford Bio-X Interdisciplinary Graduate Fellowship (E. Y. H.), and the California Institute for Regenerative Medicine (A. R. D., Grant No. RB4-06102).

\section{Citations}

[1] Paszek MJ, Zahir N, Johnson KR, Lakins JN, Rozenberg GI, Gefen A, et al. Tensional homeostasis and the malignant phenotype. Cancer Cell. 2005;8:241-54.

[2] Chaudhuri O, Gu L, Klumpers D, Darnell M, Bencherif SA, Weaver JC, et al. Hydrogels with tunable stress relaxation regulate stem cell fate and activity. Nature materials. 2016;15:326-34.

[3] Engler AJ, Sen S, Sweeney HL, Discher DE. Matrix Elasticity Directs Stem Cell Lineage Specification. Cell. 2006;126:677-89.

[4] Gilbert PM, Havenstrite KL, Magnusson KEG, Sacco A, Leonardi NA, Kraft P, et al. Substrate elasticity regulates skeletal muscle stem cell self-renewal in culture. Science (New York, NY). 2010;329:1078-81. [5] Morrissey JB, Cheng RY, Davoudi S, Gilbert PM. Biomechanical Origins of Muscle Stem Cell Signal Transduction. Journal of Molecular Biology. 2016;428:1441-54.

[6] Saha K, Keung AJ, Irwin EF, Li Y, Little L, Schaffer DV, et al. Substrate Modulus Directs Neural Stem

Cell Behavior. Biophysical Journal. 2008;95:4426-38.

[7] Guvendiren M, Burdick JA. The control of stem cell morphology and differentiation by hydrogel surface wrinkles. Biomaterials. 2010;31:6511-8.

[8] Trappmann B, Gautrot JE, Connelly JT, Strange DGT, Li Y, Oyen ML, et al. Extracellular-matrix tethering regulates stem-cell fate. Nature materials. 2012;11:642-9.

[9] Pan F, Zhang M, Wu G, Lai Y, Greber B, Schöler HR, et al. Topographic effect on human induced pluripotent stem cells differentiation towards neuronal lineage. Biomaterials. 2013;34:8131-9.

[10] Warmflash A, Sorre B, Etoc F, Siggia ED, Brivanlou AH. A method to recapitulate early embryonic spatial patterning in human embryonic stem cells. Nat Meth. 2014;11:847-54.

[11] Viswanathan P, Guvendiren M, Chua W, Telerman SB, Liakath-Ali K, Burdick JA, et al. Mimicking the topography of the epidermal-dermal interface with elastomer substrates. Integrative Biology. 2016;8:21-9.

[12] Lakins JN, Chin AR, Weaver VM. Exploring the link between human embryonic stem cell organization and fate using tension-calibrated extracellular matrix functionalized polyacrylamide gels. Methods in molecular biology (Clifton, NJ). 2012;916:317-50.

[13] Przybyla L, Lakins JN, Sunyer R, Trepat X, Weaver VM. Monitoring developmental force distributions in reconstituted embryonic epithelia. Methods (San Diego, Calif). 2016;94:101-13.

[14] Przybyla L, Lakins Johnathon N, Weaver Valerie M. Tissue Mechanics Orchestrate Wnt-Dependent Human Embryonic Stem Cell Differentiation. Cell stem cell. 2016.

[15] Bedzhov I, Zernicka-Goetz M. Self-organizing properties of mouse pluripotent cells initiate morphogenesis upon implantation. Cell. 2014;156:1032-44.

[16] Taniguchi K, Shao Y, Townshend RF, Tsai YH, DeLong CJ, Lopez SA, et al. Lumen Formation Is an Intrinsic Property of Isolated Human Pluripotent Stem Cells. Stem cell reports. 2015;5:954-62. 
[17] Shahbazi MN, Jedrusik A, Vuoristo S, Recher G, Hupalowska A, Bolton V, et al. Self-organization of the human embryo in the absence of maternal tissues. Nat Cell Biol. 2016;18:700-8.

[18] Deglincerti A, Croft GF, Pietila LN, Zernicka-Goetz M, Siggia ED, Brivanlou AH. Self-organization of the in vitro attached human embryo. Nature. 2016;533:251-4.

[19] Varelas X. The Hippo pathway effectors TAZ and YAP in development, homeostasis and disease. Development. 2014;141:1614-26.

[20] Aragona M, Panciera T, Manfrin A, Giulitti S, Michielin F, Elvassore N, et al. A Mechanical Checkpoint Controls Multicellular Growth through YAP/TAZ Regulation by Actin-Processing Factors. Cell. 2013;154:1047-59.

[21] Nishioka N, Inoue K, Adachi K, Kiyonari H, Ota M, Ralston A, et al. The Hippo signaling pathway components Lats and Yap pattern Tead4 activity to distinguish mouse trophectoderm from inner cell mass. Developmental cell. 2009;16:398-410.

[22] Musah S, Wrighton PJ, Zaltsman Y, Zhong X, Zorn S, Parlato MB, et al. Substratum-induced differentiation of human pluripotent stem cells reveals the coactivator YAP is a potent regulator of neuronal specification. Proceedings of the National Academy of Sciences of the United States of America. 2014;111:13805-10.

[23] Musah S, Morin SA, Wrighton PJ, Zwick DB, Jin S, Kiessling LL. Glycosaminoglycan-binding hydrogels enable mechanical control of human pluripotent stem cell self-renewal. ACS nano. 2012;6:10168-77.

[24] Park YD, Tirelli N, Hubbell JA. Photopolymerized hyaluronic acid-based hydrogels and interpenetrating networks. Biomaterials. 2003;24:893-900.

[25] Branco da Cunha C, Klumpers DD, Li WA, Koshy ST, Weaver JC, Chaudhuri O, et al. Influence of the stiffness of three-dimensional alginate/collagen-I interpenetrating networks on fibroblast biology. Biomaterials. 2014;35:8927-36.

[26] Chaudhuri O, Koshy ST, Branco da Cunha C, Shin J-W, Verbeke CS, Allison KH, et al. Extracellular matrix stiffness and composition jointly regulate the induction of malignant phenotypes in mammary epithelium. Nature materials. 2014;13:970-8.

[27] Aratyn-Schaus Y, Oakes PW, Stricker J, Winter SP, Gardel ML. Preparation of complaint matrices for quantifying cellular contraction. Journal of visualized experiments : JoVE. 2010.

[28] Calvet D, Wong JY, Giasson S. Rheological Monitoring of Polyacrylamide Gelation: Importance of Cross-Link Density and Temperature. Macromolecules. 2004;37:7762-71.

[29] Gelfi C, Righetti PG. Polymerization kinetics of polyacrylamide gels II. Effect of temperature. ELECTROPHORESIS. 1981;2:220-8.

[30] Claassen DA, Desler MM, Rizzino A. ROCK Inhibition Enhances the Recovery and Growth of Cryopreserved Human Embryonic Stem Cells and Human Induced Pluripotent Stem Cells. Molecular Reproduction and Development. 2009;76:722-32.

[31] Huff J. The Airyscan detector from ZEISS: confocal imaging with improved signal-to-noise ratio and super-resolution. Nat Meth. 2015;12.

[32] Carpenter AE, Jones TR, Lamprecht MR, Clarke C, Kang I, Friman O, et al. CellProfiler: image analysis software for identifying and quantifying cell phenotypes. Genome Biology. 2006;7:1-11.

[33] Efron B, Tibshirani RJ. An Introduction to the Bootstrap: Taylor \& Francis; 1994.

[34] Pless DD, Lee YC, Roseman S, Schnaar RL. Specific cell adhesion to immobilized glycoproteins demonstrated using new reagents for protein and glycoprotein immobilization. The Journal of biological chemistry. 1983;258:2340-9.

[35] Beningo KA, Lo CM, Wang YL. Flexible polyacrylamide substrata for the analysis of mechanical interactions at cell-substratum adhesions. Methods in cell biology. 2002;69:325-39.

[36] Damljanovic V, Lagerholm BC, Jacobson K. Bulk and micropatterned conjugation of extracellular matrix proteins to characterized polyacrylamide substrates for cell mechanotransduction assays. BioTechniques. 2005;39:847-51. 
[37] Caliari SR, Burdick JA. A practical guide to hydrogels for cell culture. Nat Meth. 2016;13:405-14. [38] Boudou T, Ohayon J, Picart C, Tracqui P. An extended relationship for the characterization of Young's modulus and Poisson's ratio of tunable polyacrylamide gels. Biorheology. 2006;43:721-8. [39] Ludwig TE, BergendahI V, Levenstein ME, Yu J, Probasco MD, Thomson JA. Feeder-independent culture of human embryonic stem cells. Nature methods. 2006;3:637-46.

[40] Hirate Y, Cockburn K, Rossant J, Sasaki H. Tead4 is constitutively nuclear, while nuclear vs. cytoplasmic Yap distribution is regulated in preimplantation mouse embryos. Proceedings of the National Academy of Sciences of the United States of America. 2012;109:E3389-90; author reply E91-2. [41] Cockburn K, Biechele S, Garner J, Rossant J. The Hippo pathway member Nf2 is required for inner cell mass specification. Current biology : CB. 2013;23:1195-201.

[42] Qin H, Hejna M, Liu Y, Percharde M, Wossidlo M, Blouin L, et al. YAP Induces Human Naive Pluripotency. Cell reports. 2016;14:2301-12.

[43] MacArthur BD, Ma'ayan A, Lemischka IR. Systems biology of stem cell fate and cellular reprogramming. Nature reviews Molecular cell biology. 2009;10:672-81.

[44] Hirate Y, Hirahara S, Inoue K, Suzuki A, Alarcon VB, Akimoto K, et al. Polarity-dependent distribution of angiomotin localizes Hippo signaling in preimplantation embryos. Current biology : CB. 2013;23:118194.

[45] Dobreva MP, Pereira PN, Deprest J, Zwijsen A. On the origin of amniotic stem cells: of mice and men. The International journal of developmental biology. 2010;54:761-77. 



Incubate at $37 \mathrm{C}$ for 30 minutes

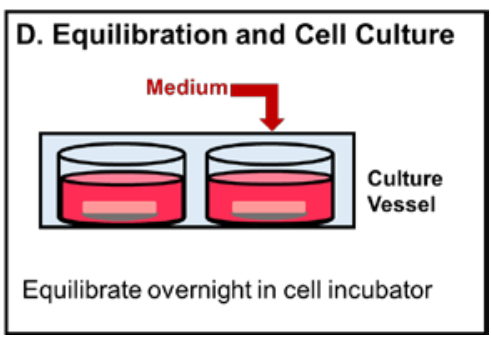

Figure 1. Preparation of semi-interpenetrating composite matrix of Matrigel and polyacrylamide. (A) A precursor solution is made by combining a stock of acrylamide and bis-acrylamide monomer 1:1 with a freshly thawed Matrigel aliquot. Components are kept on ice at all times. The mixture is degassed for 30 minutes. (B) Ammonium persulfate (APS) and tetramethylethylenediamine (TEMED) are added to the precursor solution and the mixture is immediately sandwiched between an aldehyde-functionalized coverslip and a passivated (hydrophobic) glass slide. (C) After polyacrylamide polymerization, gelation of Matrigel is performed by transferring the sandwiched gel into a humidified $37^{\circ} \mathrm{C}$ incubator. (D) The resulting composite matrix is equilibrated overnight in Dulbecco's Modified Eagle medium (DMEM) in a humidified cell culture incubator before cell seeding. 

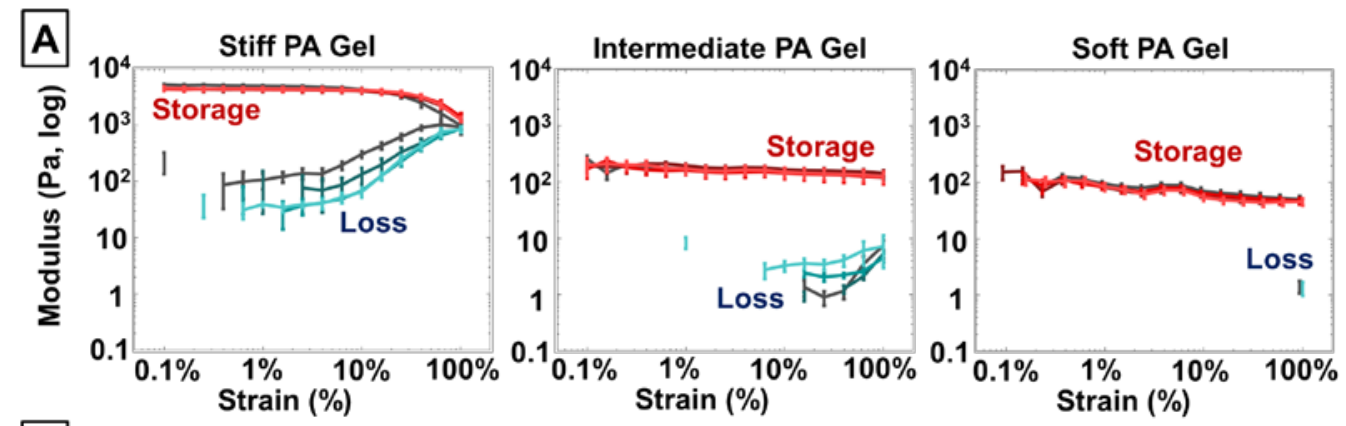

Storage Modulus (G')
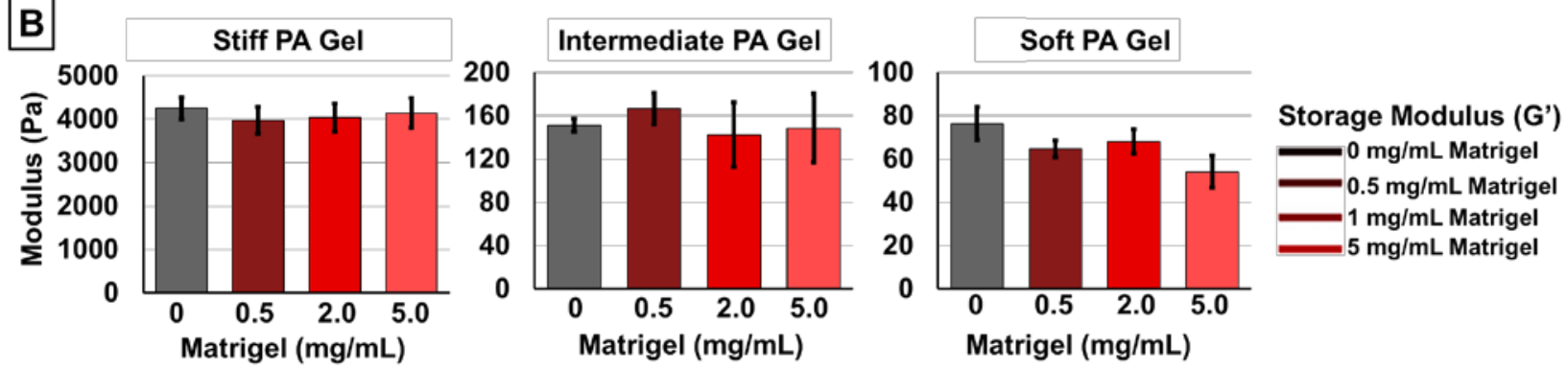

Figure 2. Shear modulus of composite matrix. (A) Log-log plot of the shear elastic storage ( $\left.G^{\prime}\right)$ and loss (G") moduli measured by an oscillating parallel plate at $0.25 \mathrm{~Hz}$ from $0.1 \%$ to $100 \%$ strain; instrument resolution limited measurements on the intermediate and soft composite matrixes. Four concentrations of Matrigel $-0 \mathrm{mg} / \mathrm{mL}, 0.5 \mathrm{mg} / \mathrm{mL}, 2 \mathrm{mg} / \mathrm{mL}$, and $5 \mathrm{mg} / \mathrm{mL}$ - were measured for each of three PA formulations - stiff PA ( $7.5 \%$ acrylamide, $0.1 \%$ bis-acrylamide), intermediate PA (3\% acrylamide, 0.06\% bis-acrylamide), and soft PA (3\% acrylamide, $0.04 \%$ bis-acrylamide). All curves show the mean and standard error on the mean from either 15 measurements (for all soft PA gels) or 8 measurements (for all intermediate and stiff PA gels) collected from at least three independent preparation and measurement sessions. When measurable, the loss modulus was more than 10 times smaller than the storage modulus at $\leq 10 \%$ strain for the stiff PA gel and all strains for the intermediate PA gel, indicating a highly elastic response. For the softest hydrogel, the only measurable loss modulus was $\sim 1.3 \mathrm{~Pa}$ at $100 \%$ strain, more than 30 times smaller than the storage modulus of $\sim 45 \mathrm{~Pa}$ at the same strain. (B) The storage modulus plotted in linear space for each condition allows comparison of the physical properties at different dilutions of Matrigel. The storage modulus at $10 \%$ strain is plotted for all conditions. The addition of $5 \mathrm{mg} / \mathrm{mL}$ Matrigel $(0.5 \% \mathrm{w} / \mathrm{v})$ into the polyacrylamide matrix appeared to have a slight softening effect on the softest PA material, but the effect was not statistically significant $(p=0.07)$. Matrigel addition did not significantly change the shear moduli of the other conditions. 


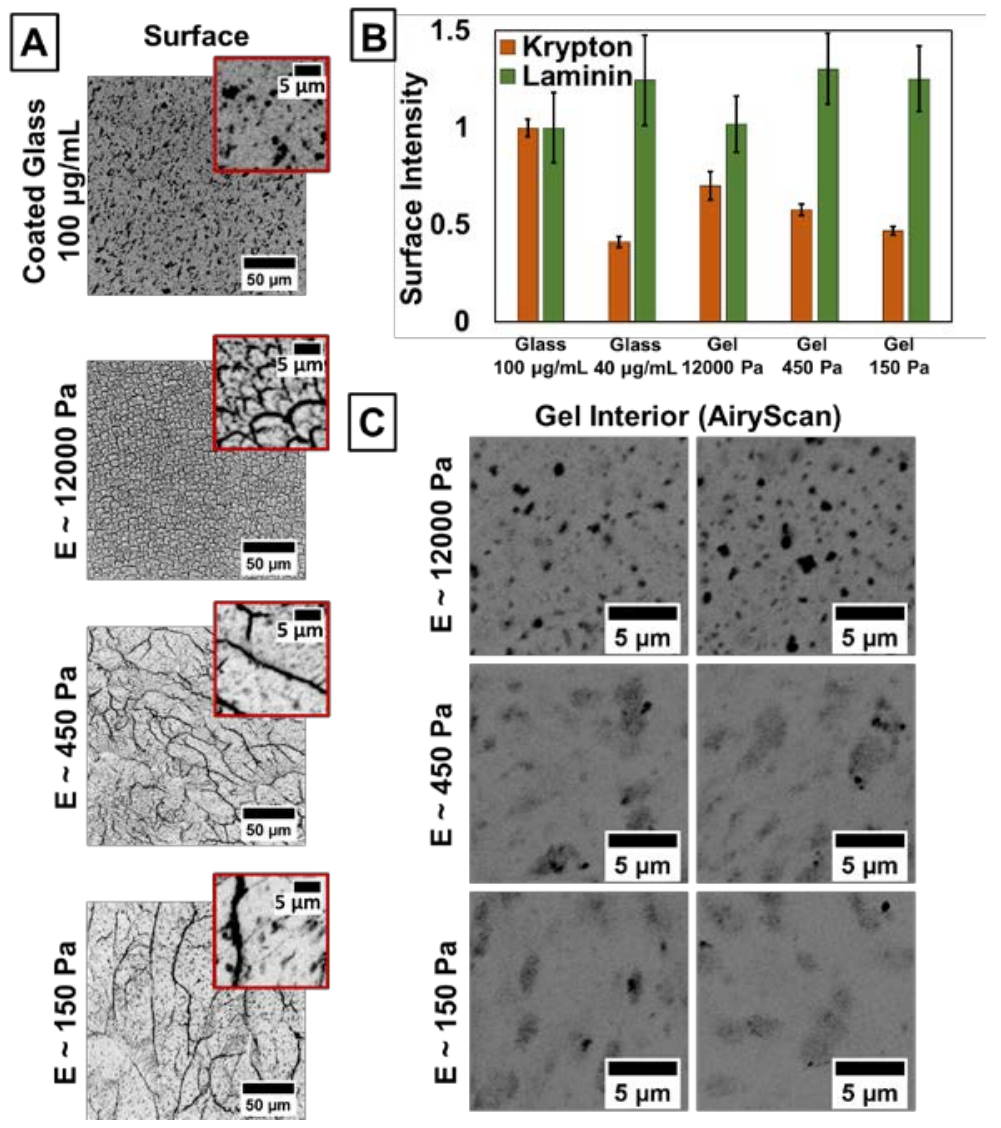

Figure 3. Protein distribution within composite matrices. (A) A confocal slice showing the surface distribution of protein embedded within the PA/Matrigel composite visualized with Krypton Orange fluorescent protein stain (Inverted Gray colormap, black indicates high intensity). (B) Average and standard error on the mean for each condition as measured using confocal microscopy. Krypton Orange stain: $n=7,27$ imaged positions total for each of the three semi-IPN conditions and the $100 \mu \mathrm{g} / \mathrm{mL}$ Matrigel coated glass, $\mathrm{n}=5,17$ imaged positions total for the $40 \mu \mathrm{g} / \mathrm{mL}$ Matrigel coated glass. The mean intensity of the Krypton stain was not significantly different between the $E \sim 12000 \mathrm{~Pa}$ gel and the $E \sim 450$ $\mathrm{Pa}$ gel $(p=.12)$ but was significantly different for the $\mathrm{E} \sim 150 \mathrm{~Pa}$ gel $(p=.005)$. Laminin stain: $n=2,10$ imaged positions total for all conditions. The mean intensities for all laminin conditions were statistically indistinguishable from each other and the glass control. (C) A super-resolved AiryScan fluorescence image shows the distribution of protein within the PA/Matrigel composite at high resolution. Two representative images from different hydrogels are shown (Inverted Gray colormap). 

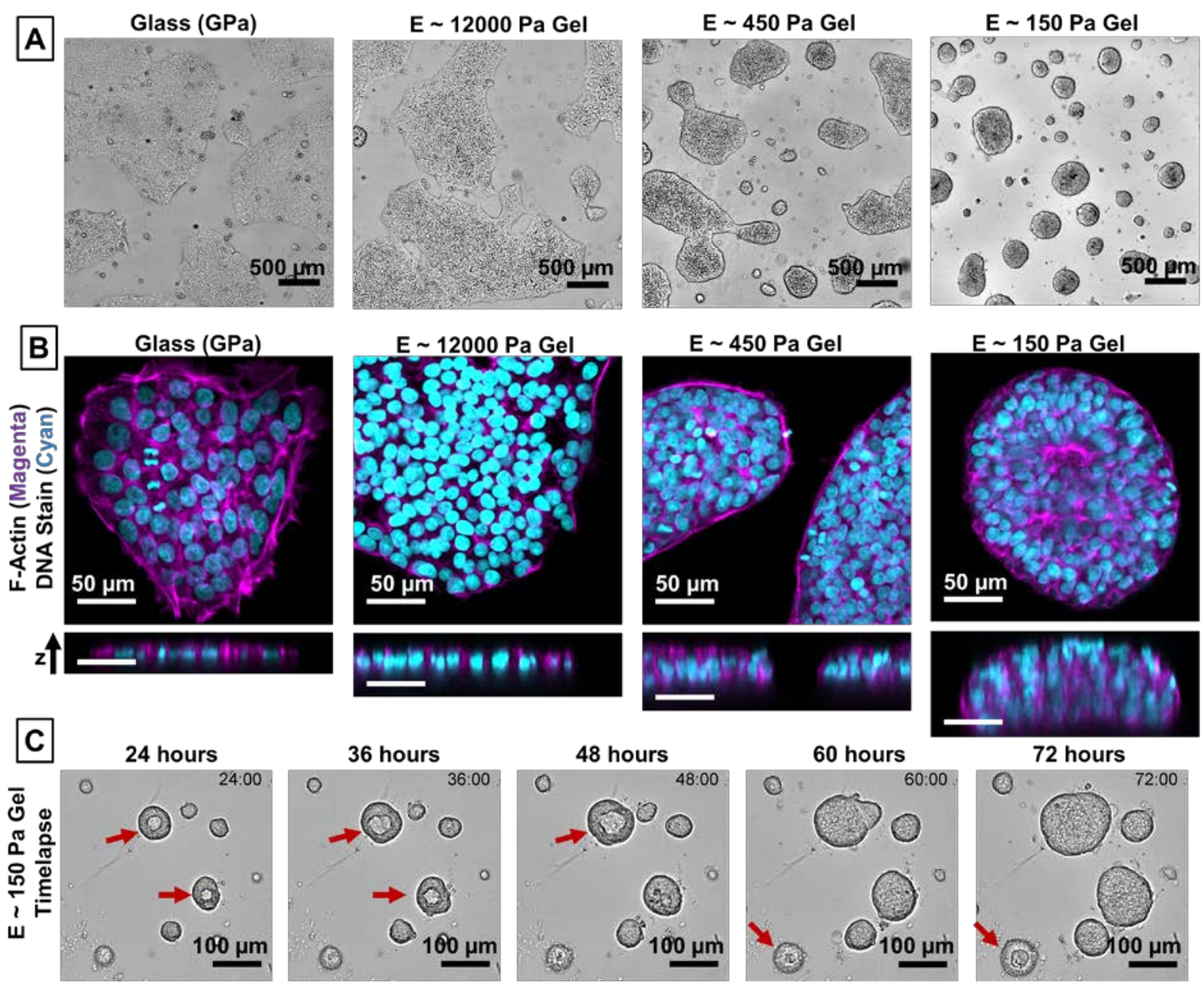

Figure 4: Colony morphology on composite substrates. As the composite gel stiffness decreases, colonies become more rounded and multilayered. (A) Phase-contrast image of human embryonic stem cell (WA09) colonies cultured on composite PA/Matrigel hydrogels. Colonies shown here were cultured on composite hydrogels for a total of eight days, having been sub-cultured on day four as described in Methods 2.4. (B) A set of colonies cultured for five days on composite hydrogels were stained with fluorescent phalloidin (F-Actin, magenta) and Hoechst (DNA Stain, cyan). Images were median filtered with ImageJ (2 pixel radius) to reduce noise. A single confocal slice is shown with a corresponding orthogonal slice. (C) A time-lapse frame capture of human embryonic stem cell colonies (WA09) grown on a soft $\mathrm{E} \sim 150 \mathrm{~Pa}$ composite gel 24 hours post seeding. Transient colony dynamics with ring-like cell density around colony edge are indicated (red arrows). 


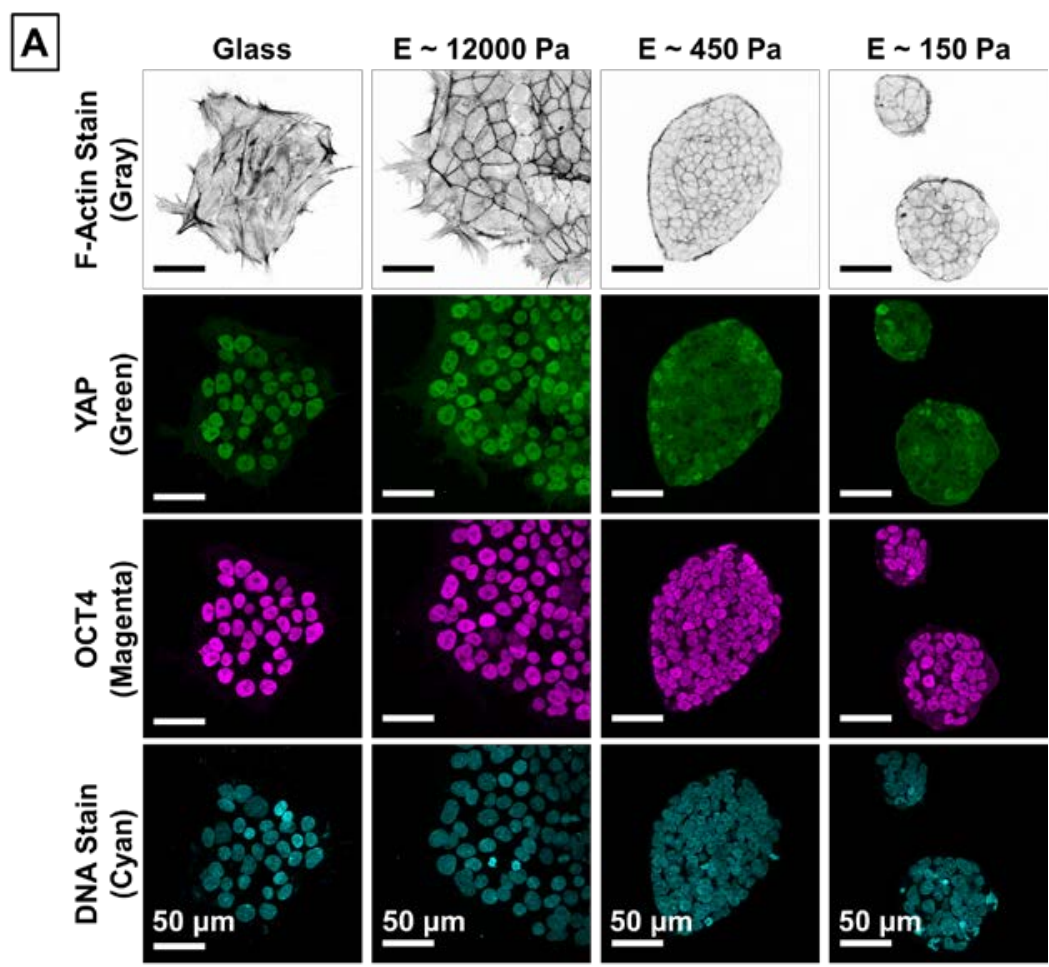

B 1
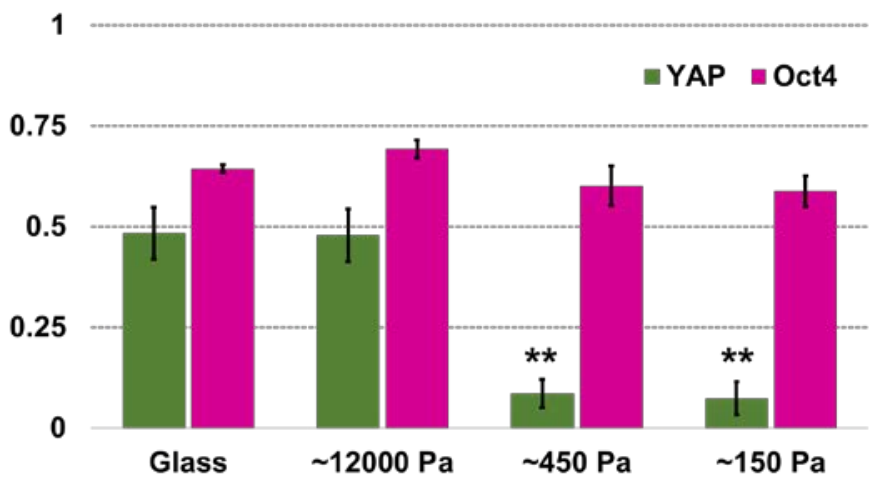

Figure 5. YAP localization. (A) Fluorescent images of pluripotent cell colonies grown on composite Matrigel/PA hydrogels for five days. Here, maximum projections of three confocal slices at the colonymatrix interface show changes in F-actin structure, the localization of the YAP transcriptional coactivator, and the pluripotency marker Oct4 as a function of surface stiffness. (B) Pearson's correlation coefficient averaged across all voxels within the colony for the entire 3D stack. Decreased localization of YAP to the nucleus is shown by a decrease in the correlation of YAP signal with the DNA stain (Hoescht). The localization of YAP is significantly decreased on the $\mathrm{E} \sim 450 \mathrm{~Pa}$ and $\mathrm{E} \sim 150 \mathrm{~Pa}$ composite substrates (** indicates $p<0.01$ ) relative to Glass, while the localization of Oct4 is not significantly changed between these conditions. 
A
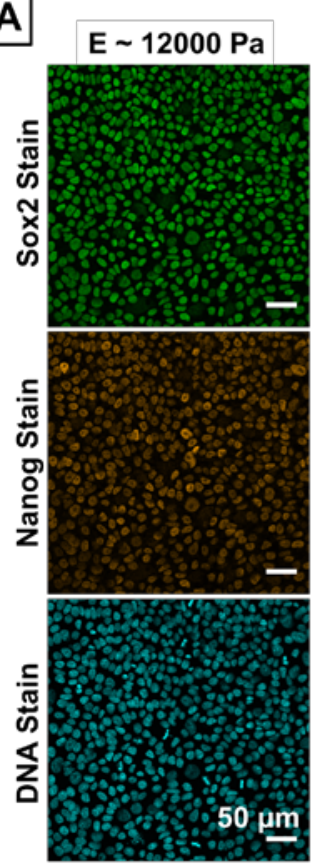
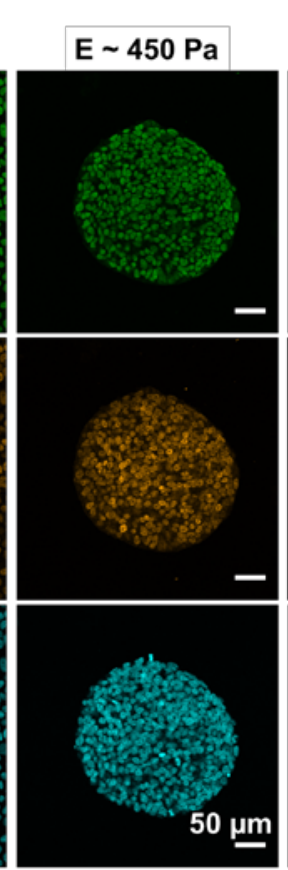

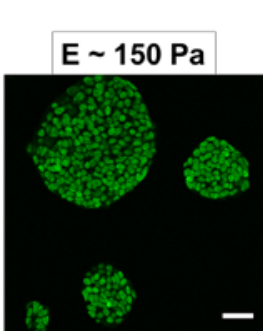

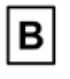
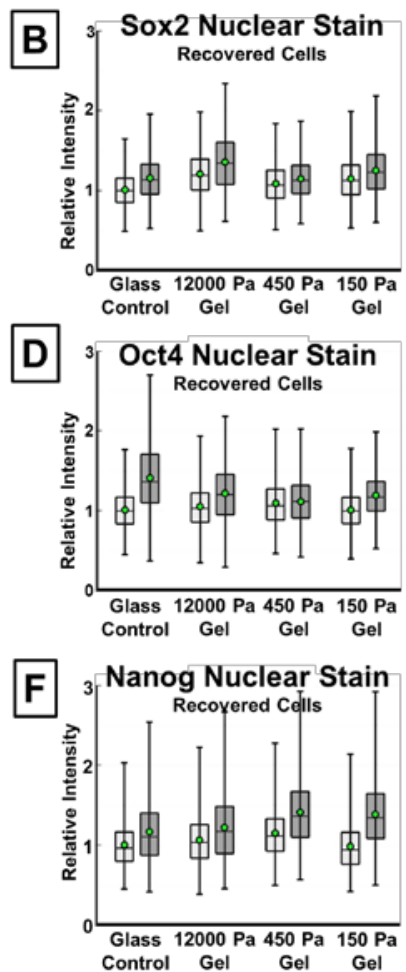
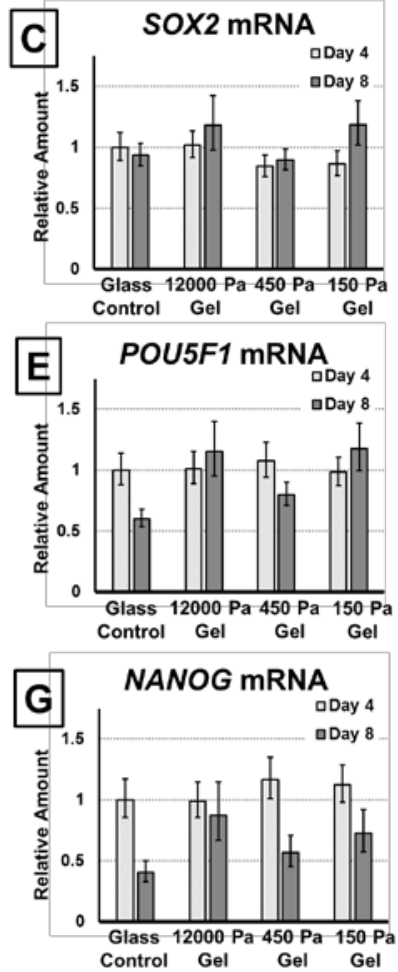

Figure 6. Maintenance of pluripotency. (A) Pluripotency factor localization on composite substrate. Example confocal slices through pluripotent cell colonies cultured on a hydrogel substrates for five days show clear nuclear stains of the transcription factors Sox2 and Nanog. (B, D, F) Pluripotency staining quantification of recovered cells shows no irreversible changes in pluripotency. hESCs (WA09) were seeded onto composite gels of different stiffness and a Matrigel-coated glass control. After four days, cells were detached from the substrates and seeded onto a glass coverslip prior to fixation and immunostaining (see schematic in Figure S5). Recovered cells were seeded onto coated glass for two days prior to fixation, required for segmentation and quantitative staining. Intensity distribution of the segmented nuclei relative to a Day 4 glass control is plotted, normalized to a control DNA stain (Hoechst). Boxplots show the intensity distribution with whiskers indicating the $1^{\text {st }}$ and $99^{\text {th }}$ percentile, a central line at the median value, and a green dot indicating the average value within the $1^{\text {st }}$ and $99^{\text {th }}$ percentile. Each plot represents at least 3000 nuclei (average $n=10365$ ) from a total of three staining sets across two independent experiments. (C, E, G) Pluripotency factor transcription is maintained on composite substrates. As a complement to immunostaining, mRNA was directly extracted from cells on composite hydrogels for qPCR analysis; each bar represents five measurements in two independent experiments. 

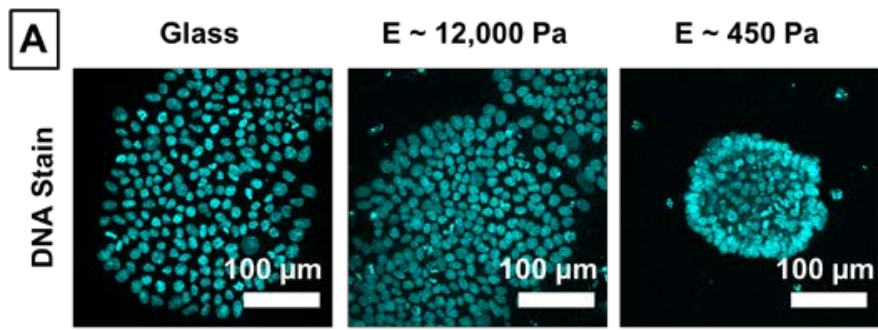

$\mathrm{E} \sim 150 \mathrm{~Pa}$
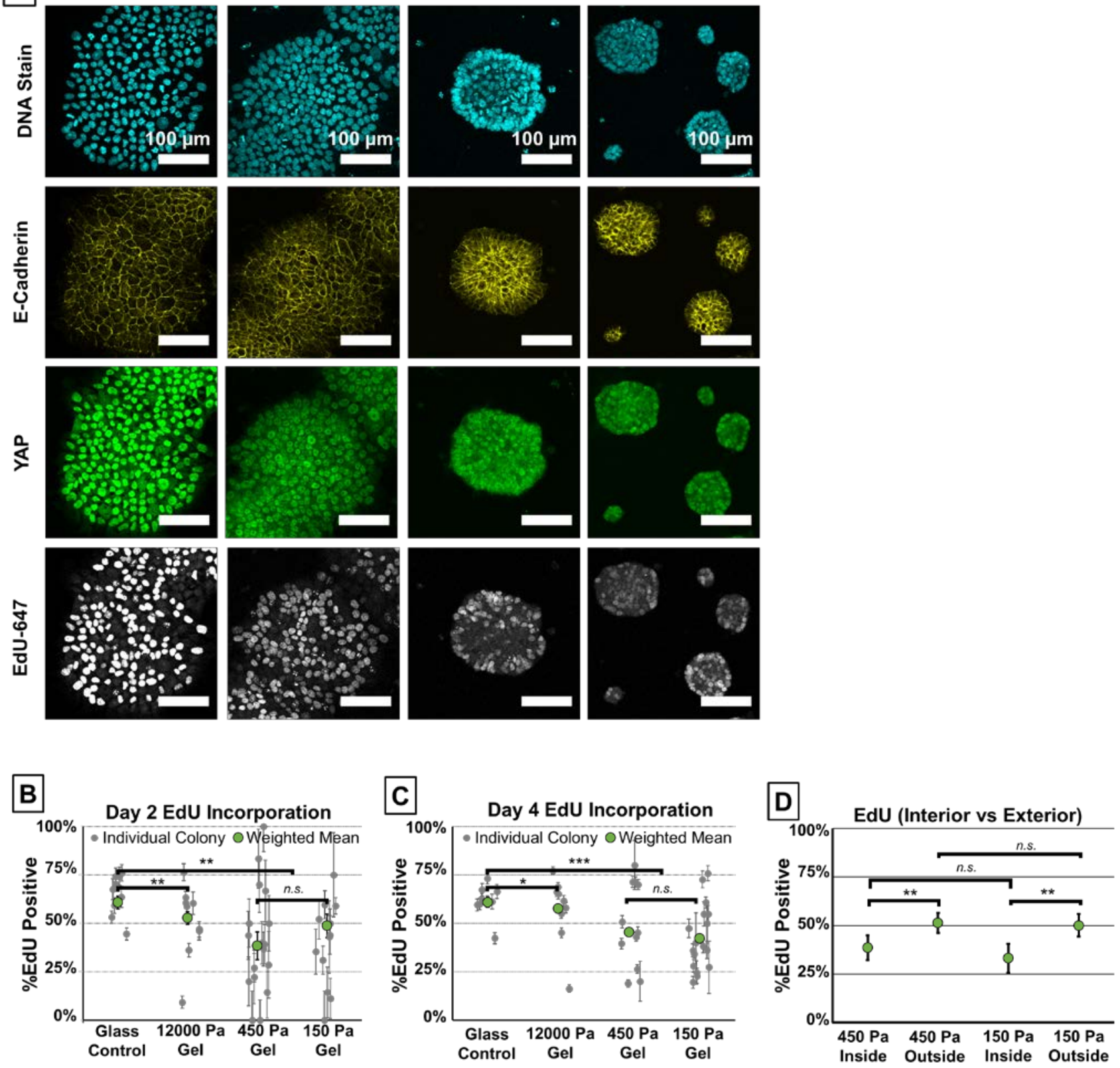

\section{E}
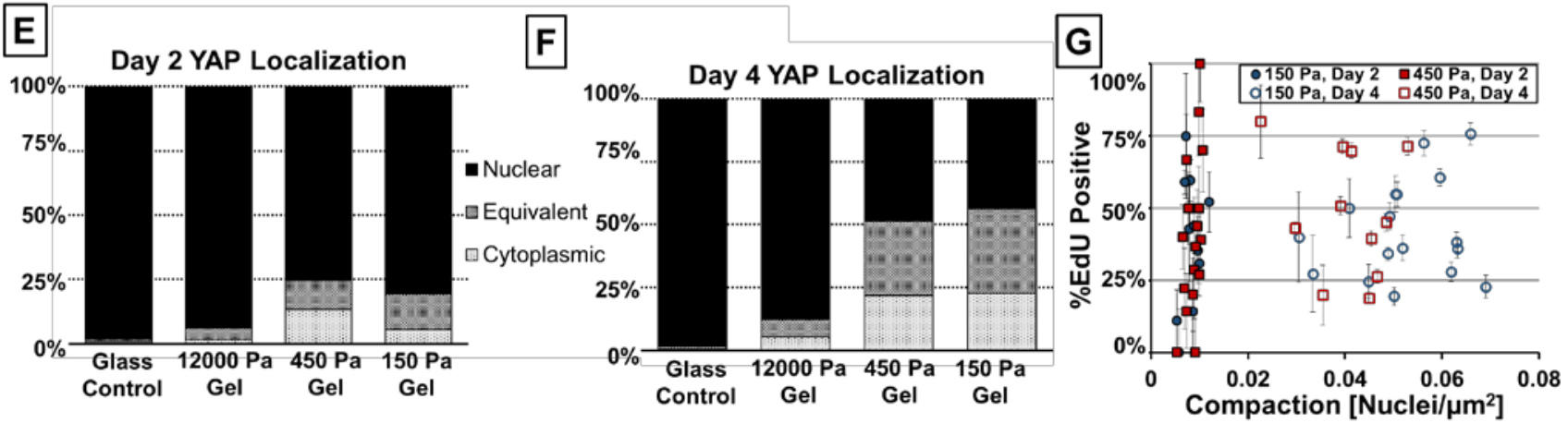
Figure 7. Proliferation in multilayered pluripotent cell colonies. (A) Example confocal images of WA09 hESCs cultured on hydrogel substrates for four days. Cells were pulse-labeled with EdU and immunostained for DNA, E-Cadherin and YAP. (B) The fraction of EdU-positive cells after two days of culture on the hydrogel; gray points show the fraction positive within each colony with error bars indicating the standard error on the mean from bootstrap analysis (see Methods 2.7). The weighted average (green) is plotted with a $98 \%$ confidence interval. ${ }^{*} p<0.05$, ${ }^{* *} p<0.01$. (C) Fraction of EdU-positive cells after four days of culture on the hydrogel, plotted as in (B). (D) Fraction of EdU-positive cells on the inside versus outside of individual colonies after four days of culture. Cells within $10 \mu \mathrm{m}$ of the edge were labeled "outside" and the cell more than $10 \mu \mathrm{m}$ from the colony edge were labeled "inside" in a midplane slice. Because the colony structure of the $\mathrm{E} \sim 12000 \mathrm{~Pa}$ and glass control were two-dimensional (see Figure 4) only the $\mathrm{E} \sim 150 \mathrm{~Pa}$ and $450 \mathrm{~Pa}$ substrates had interior cells that were surrounded by other cells $\left({ }^{* \star} p<0.01,{ }^{* \star *} p<0.001\right)$. (E) YAP localization after two days of culture, plotted relative to the average cytoplasmic intensity in each condition. Cells with nuclei showing $<0.9$ of the cytoplasmic value were scored as having predominantly cytoplasmic YAP localization (White), while those with $>1.1$ of the cytoplasmic value were scored as having predominantly nuclear YAP localization (Black). All other nuclei were marked as "equivalent" (Gray). (F) YAP localization to the cytoplasm increased after four days of culture on the $\mathrm{E} \sim 150 \mathrm{~Pa}$ and $\mathrm{E} \sim 450 \mathrm{~Pa}$ substrates, plotted as in (E). (G) After four days of growth, the number of cells in each colony increased rapidly, but the projected colony radius grew relatively slowly due to the multilayered morphology. To test whether the increased multilayered morphology correlates with a decreased proliferation rate, the proliferation rate for individual colonies ( $>2$ cells) is plotted against the colony radius. While compaction increased significantly from day 2 to day 4 , the EdU incorporation frequency was not significantly changed. 